\title{
Eins, zwei, drei - ritsche, ratsche, rei Über die Unverzichtbarkeit eines Dritten
}

Kommentar zum Artikel von Vera Saller

\section{Christian Hauser (Zürich)}

Gebräuchlich ist die Vorgehensweise des theoriegeleiteten Denkens, alte Gedankengebäude von hohem Rang dafür zu benutzen, die genuin eigenen Gedankengänge einzuordnen und zu fundieren.

Es ist unbestritten von Relevanz, sich zu fragen, worin die Unterschiede und Gemeinsamkeiten zwischen habituellem und dynamischem Unbewussten bestehen, hilfreich auch ist eine schärfende Klärung des «Impliziten ${ }^{1}$ gegenüber dem Unbewussten in der Übertragungsebene. Ebenso ist die Frage nach der Entstehung des Denkens nicht bloss eine psychologische, sondern seit alters her auch eine philosophische.

Aber hier sei die Frage erlaubt, inwiefern eine erneute Rezeption der semiotischen und erkenntnistheoretischen Überlegungen von Charles Sanders Peirce, die erst noch gut 110 Jahre zurückliegen, besonders im Kontext der Psychoanalyse erhellend und erkenntniserweiternd sein können. Ein Rückgriff auf die kategoriale Unterscheidung zwischen Firstness, Secondness und Thirdness bei Peirce, ein Rückgriff, der sich für die Psychoanalyse als fruchtbringend erweisen soll, muss sich erst noch legitimieren.

Wenn Saller die Kategorie der Erstheit bei Peirce mit dem Primärvorgang bei Freud in Verbindung bringt, ist dies durchaus plausibel. Bei Peirce ist die Erstheit ein Sein von etwas ohne jeglichen Bezug zu oder auf etwas anderes, unmittelbare Empfindungsqualität, pure Gegenwärtigkeit, gänzlich unabhängig von der Existenz eines Objekts. Es ist ein Sein an sich, das als reine Möglichkeit besteht. Womöglich gilt auch für die Peirce'sche Kategorie der Erstheit wie auch für Freuds Begriff des Unbewussten, dass beispielsweise die lineare Zeit, die Negation, der logische Widerspruch und der Satz vom ausgeschlossenen Dritten darin keine Gültigkeit haben.

Bei den drei relationalen Kategorien von Peirce fällt einem unweigerlich das Hegelsche Dreigespann der Dialektik ein. Man könnte sich in der Tat fragen, ob das umfassende Unbewusste Freuds nicht ebenso im Kontext der Hegel'schen 
Phänomenologie des Geistes als reines Dieses verstanden werden könnte. Letzterer Einfall läuft darauf hinaus, das es auch bei den Peirce'schen Kategorien der Erstheit, Zweitheit und Drittheit darum geht, wie sich die drei zueinander verhalten und auseinander ergeben. Ich bin mit vielen anderen überzeugt, dass man die Peirce'sche Triade - beispielsweise in der semiotischen Lesart des Ikons, Index und Symbols nur unzureichend versteht, wenn man sie nicht dialektisch zu verstehen versucht. ${ }^{2}$ Desgleichen ist es bei Freud unumgänglich, die Dyade (Zweitheit) zu überwinden und - Hegelsch gesprochen - in der Dreiheit aufzuheben.

In einer semiotischen Ontologie wie der Peirce'schen vermittelt das Zeichen als repräsentierendes Symbol. Die Erstheit, also das was so ist, wie es ist, unabhängig von etwas anderem, ungebunden, wird durch ein Zweites überhaupt erst in Beziehung gesetzt oder verbunden und schliesslich durch ein Drittes in einer Repräsentation vermittelt (Triangulierung). Auf Letzteres bezugnehmend stimme ich mit Sallers Ausführungen überein, dass das dynamische Unbewusste Freuds bereits in einem Übergang zur Zweiheit anzusiedeln ist, in dem unbewusste, ambivalente Vorstellungen und Affekte unvermittelt nebeneinander bestehen. Das dynamische Unbewusste ist wohl «als kleiner Teil des umfassenderen Unbewussten» (Saller, in diesem Heft, S. 12) zu verstehen. Geradeso bin ich einer Meinung, dass «das Freud'sche Unbewusste [sich] auf den Ebenen des First und Second wiederfindet» (Saller, in diesem Heft, S. 18). Beachten wir bei diesen Überlegungen, dass das, was wir in der Psychoanalyse gemeinhin als das Unbewusste bezeichnen, ungefähr 94 Prozent unserer geistigen Tätigkeit einschliesst.

Vergegenwärtigen wir uns den besonderen Kontakt der projektiven Identifikation, wie wir ihm gelegentlich in Analysen und Therapien begegnen und oft auch analytisch nutzen, so wäre dies - in den Peirce'schen Kategorien eine nicht symbolische, affektive Kommunikation auf der Ebene des First und des Second. Das Second kommt in der projektiven Identifikation deshalb zum Zuge, weil eine projektive Identifikation bereits eine Gerichtetheit ${ }^{3}$ - wenn auch unbewusste - enthält, die es beim First nicht gibt. Ergänzend zu Vera Sallers Befund «Das Second ist der Moment, wo ein Reiz aus der Aussenwelt [...] aufschreckt» (Saller, in diesem Heft, S. 18) ist zu bemerken, dass der Reiz ebenso gut aus der Innenwelt bzw. dem Unbewussten stammen kann.

Eine längere Bemerkung zu Sallers Unterscheidung zwischen neurotischer und normaler Beharrlichkeit (Saller, in diesem Heft, S. 22) will ich noch anfügen: Das Gehirn wäre bei weitem überfordert, wenn es die Unmengen von Signalen nicht irgendwie selektionierte. Dies ist die eigentliche Grundvoraussetzung, ohne die das System Gehirn gleichsam in der Flut der Empfindungen unterginge. Man 
muss also annehmen, dass es wohl sensorische, hirnphysiologische und auch kognitive oder psychische Grenzen geben muss. In anderen Worten: Es sind einerseits die Reizschwellen, mit denen in der neuronalen Übermittlung ein Filter gesetzt ist und andererseits besteht ein weiterer Filter auf dem Weg der Bedeutungen zum Bewusstsein; man könnte einmal von einem Reizfilter, im letzteren Fall von einem Inhaltsfilter sprechen. Die Psychose und der Schock sind Beispiele dafür, was beim Ausfall des Filters geschehen kann:Wenn man maximal aktiviert ist oder etwas Schreckliches erlebt hat, ist man nicht mehr imstande, die Aufmerksamkeit selektiv anzuwenden. Man empfindet kleinste, unwichtige Details, wird von diesen gleichsam überschwemmt, was auf Kosten rational-kohärenter Gedanken und koordinierter Handlung geht.

Ein ähnliches Phänomen zeigt sich auch in unserer medialen Kultur. Man spricht davon, dass wir heute einem informational overload ausgesetzt seien. So intendieren die schnellen Schnitte in Videoclips, Filmen und Fernsehsendungen, dass der Zuschauer durch wiederholte, schnell folgende Reize gefesselt bleibt. Doch diese ständige Überraschung geht wohl auf Kosten der Aufmerksamkeit, die sich nicht mehr auf bestimmte Inhalte zu konzentrieren vermag.

Die selektive Wahrnehmung reicht von der lebensnotwendigen, natürlichen Selektion für das Bewusstsein über den so genannten «perceptual-defence-Effekt» bis hin zur Verdrängung und Verleugnung. Alle drei Stufen stehen gleichsam unter einem ökonomischen Prinzip: Das Bewusstsein soll in einem funktionstüchtigen Gleichgewicht gehalten werden.

Die Referenz auf Charles Sanders Peirce schliesslich hat also durchaus das Ziel, der Drittheit sowohl entwicklungspsychologische als auch erkenntnistheoretische Geltung zuzugestehen. Das logische und semiotische Dreigespann ist keine sensualistische Steigerung, sondern eine dynamisch-dialektische Einheit. Ohne ein Drittes - und sei es bloss eine Symbolisierung - kann beispielsweise «die Mutter ihre spiegelnde Funktion» (Saller, in diesem Heft, S. 21) nicht übernehmen. Konflikte auf der Ebene der Zweiheit werden dank dem Dritten bzw. dank einer gelungenen Symbolisierung aufgehoben.

\section{Anmerkungen}

1 Im Sinne der Mikroprozesse der Bostoner Change Process Study Group (CPSG). Boston CPSG reports I and II; besonders D. N. Stern et al. (1998), Non-interpretive mechanisms in psychoanalytic therapy: The something more than interpretation. International Journal for Psychoanalysis, 79, S. 908-921.

$2 \quad$ Ch.S. Peirce, CP 1.451: "Were I to take the categories of First, Second, and Third, and to classify in their order all the forms of secondness and then all those of thirdness, connecting each with the preceding by a process of transition, [...] I should be developing 
my doctrine as it seems to me very much in the spirit and method in which Hegel develops his Encyclopaedia." (The Collected Papers of Charles Sanders Peirce, eds. C. Hartshorne, P. Weiss and A. Burks, Cambridge, Harvard University Press).

3 Ich ziehe hier den deutschen Ausdruck dem der Intentionalität ausdrücklich vor. 